\title{
ON POWERS OF NON-NEGATIVE MATRICES
}

\author{
JOHN C. HOLLADAY AND RICHARD S. VARGA ${ }^{1}$
}

Let $A=\left\|a_{i, j}\right\|$ be an $n \times n$ matrix consisting of non-negative elements. It is well known $[1, \mathrm{p} .463]$ that $A$ is primitive if and only if, for some positive integer $n, A^{n}$ has all its elements positive. One needs to know only this property of primitive matrices to understand this paper. If $A^{k}$ is positive (i.e. has all its elements positive), then $A^{h}$ is also positive for all integers $h>k\left[1\right.$, p. 463]. ${ }^{2}$ Letting $A$ be primitive, we shall define $\gamma(A)$ as the smallest positive integer $h$ such that $A^{h}$ is positive.

Wielandt $\left[2\right.$, p. 648] stated without proof the inequality ${ }^{3}$

$$
\gamma(A) \leqq n^{2}-2 n+2,
$$

and gave an example to show that $\gamma(A)$ could equal $n^{2}-2 n+2$. In the special case that all the diagonal elements of $A$ are positive, Wielandt [2, p. 644] showed that one may obtain the better bound

$$
\gamma(A) \leqq n-1 .
$$

In this paper, we show that when there are one or more positive diagonal elements of $A$ (or of one of its low order powers), bounds may be found for $\gamma(A)$ which are better than (1), although not necessarily as good as (2). We shall also give an easy proof of (1).

In our discussion, we shall assume that the matrix $A$ is non-negative and primitive. ${ }^{4}$ Let $J$ be the set of positive integers one through $n$. For $L$ a subset of $J$, define $F^{0}(L)=L$ and, by induction, for $h$ a positive integer, define $F^{h}(L)$ as the set of all $i \in J$ such that for some $j \in F^{h-1}(L), a_{i, j}>0$. For $h$ a non-negative integer, and $j \in J$, define $F^{h}(j)$ as $F^{h}(L)$ where $L$ is the set containing $j$ and only $j$. We remark that, for $h$ a positive integer, the element of $A^{h}$ in the $i$ th row and $j$ th column is positive if and only if $i \in F^{h}(j)$.

Lemma 1. $F(J)=J$.

Received by the editors January 16, 1958.

1 Work done under the auspices of the A.E.C.

${ }^{2}$ One may also use Lemma 1 of this paper.

${ }^{3}$ Others, in examining the fundamental properties of non-negative primitive matrices have indirectly obtained bounds for $\gamma(A)$. For example, as pointed out by Wielandt [2, p. 647], Frobenius [1, p. 463] indirectly obtained the bound $2 n^{2}-2 n$, while Herstein $\left[3\right.$, p. 20] indirectly obtained the bound $n^{n}$ for $\gamma(A)$.

4 This obviously implies that $A$ is irreducible. See $[1$, p. 463]. 
Proof. For $j \in J, J=F^{\gamma(A)}(j) \subseteq F^{\gamma(A)}(J)=F^{\left(F^{\gamma(A)-1}(J)\right] \subseteq F(J) \subseteq J}$.

LeMma 2. If $L$ is a proper subset of $J$, then $F(L)$ contains some element not in $L$.

Proof. If not, then $J \supseteq L \supseteq F(L) \supseteq \cdots \supseteq F^{\gamma(A)}(L)=J$ which contradicts $J \neq L$.

COROLlary. If $h \leqq n-1$, then $\{j\} \cup F(j) \cup \ldots \cup F^{h}(j)$ contains at least $h+1$ elements.

Proof. This is obviously true for $h=0$. Using mathematical induction, assume it is true for some $0 \leqq h \leqq n-1$. Set $L=\{j\} \cup \cdots \cup F^{h}(j)$, and apply Lemma 2 .

We remark that, given $j \in J$, the set of integers $h$ such that $j \in F^{h}(j)$ is a semigroup. Therefore, properties described below may be easily observed by observing the first few iterates of $A$.

Lemma 3. Let $k$ be a non-negative integer, and $j \in J$. For $h \geqq k$, let $j \in F^{h}(j)$. Then, $F^{n-1+k}(j)=J$.

Proof. The corollary above implies that $\{j\} \cup \cdots \cup F^{n-1}(j)=J$. For each $0 \leqq h \leqq n-1, j \in F^{n-1+k-h}(j)$, and so $F^{h}(j) \subseteq F^{n-1+k}(j)$. Therefore, $J=\bigcup_{h=0}^{n-1} F^{h}(j) \subseteq F^{n-1+k}(j) \subseteq J$.

TheOREM 1. Let $k$ be a non-negative integer. Let there be at least $d>0$ elements $j$ of $J$ such that for $h \geqq k$, the jth diagonal element of $A^{h}$ is positive. Then, $\gamma(A) \leqq 2 n-d-1+k$.

Proof. The corollary above implies that, for each $j \in J$, there exists $0 \leqq h \leqq n-d$ such that $F^{h}(j)$ contains at least one of the $d$ elements described above. Then,

$$
J \supseteq F^{2 n-d-1+k}(j)=F^{n-d-h}\left\{F^{n-1+k}\left[F^{h}(j)\right]\right\} \supseteq F^{n-d-h}(J)=J .
$$

Corollary. Let at least $d>0$ of the diagonal elements of $A$ be positive. Then, $\gamma(A) \leqq 2 n-d-1 .^{5}$

Theorem 2. Let $h$ be a positive integer, and let $A+A^{2}+\cdots+A^{h}$ have at least $d>0$ of its diagonal elements positive. Then, $\gamma(A) \leqq n-d$ $+h(n-1)$.

Proof. Let $j$ be one of the $d$ elements such that $j \in F^{p}(j)$ for some $p, 1 \leqq p \leqq h$. Then, if we substitute 0 for $k$, and $F^{p}$ for $F$, we may apply Lemma 3, and conclude that $F^{(n-1) p}(j)=J$. Choose arbitrarily $j^{\prime} \in J$. Then, the corollary to Lemma 2 implies that there exists an $l$,

5 If all the diagonal elements of $A$ are positive, then $d=n$, and the inequality of the corollary reduces to Wielandt's result (2). 
$0 \leqq l \leqq n-d$ such that $F^{l}\left(j^{\prime}\right)$ contains at least one of these $d$ elements. Therefore, $\quad J \supseteq F^{n-d+h(n-1)}\left(j^{\prime}\right)=F^{n-d-l+(h-p)(n-1)}\left\{F^{p(n-1)}\left[F^{l}\left(j^{\prime}\right)\right]\right\}$ $\supseteq F^{n-d-l+(h-p)(n-1)}(J)=J$, since $n-d-l+(h-p)(n-1) \geqq 0$.

Corollary. Let $A$ be non-negative and positively symmetric in that $a_{i, j}>0$ if and only if $a_{j, i}>0$. Then, $\gamma(A) \leqq 2(n-1)$.

Proof. $A^{2}$ has all its diagonal elements positive. Now, apply Theorem 2.

TheOREM 3. $\gamma(A) \leqq n^{2}-2 n+2$.

Proof. Given $j \in J$, consider the case where $\{j\} \cup \cdots \cup F^{n-2}(j)$ $\neq J$. Then, for $1 \leqq h \leqq n-1, F^{h}(j)$ contains exactly one element not in $\{j\} \cup \ldots \cup F^{h-1}(j)$. Let $p$ be the smallest positive integer such that $F^{p}(j)$ contains at least two elements. Then, there exists an integer $m<p$ such that $m>0$ (unless $p=1$, in which case $m=0$ ) and such that $F^{m}(j) \subseteq F^{p}(j)=F^{m+(p-m)}(j) \subseteq F^{m+2(p-m)}(j) \subseteq \cdots$. Lemma 2 implies that $F^{m+(n-1)(p-m)}(j)=J$. But $p \leqq n$ implies that

$$
m+(n-1)(p-m)=p+(n-2)(p-m) \leqq n^{2}-2 n+2 .
$$

If $\{j\} \cup \cdots \cup F^{n-2}(j)=J$, then there exists an integer $h, 0 \leqq h$ $\leqq n-1$, such that $F^{0}(j) \subseteq F^{h}(j) \subseteq \cdots \subseteq F^{(n-1) h}(j)=J$. But, $(n-1) h$ $\leqq n^{2}-2 n+1<n^{2}-2 n+2$. This completes the proof.

Let $A$ and $B$ be two non-negative primitive matrices such that if $A=\left\|a_{i, j}\right\|$, and $B=\left\|b_{i, j}\right\|$, then $a_{i, j}>0$ implies that $b_{i, j}>0$. It is clear that $\gamma(A) \geqq \gamma(B)$. Furthermore, if $B$ has many positive elements for which there are no corresponding positive elements of $A$, then one would expect to have $\gamma(A)>\gamma(B)$. We shall show that when there are many positive off-diagonal elements of a non-negative primitive matrix, some of the preceding inequalities may be improved.

Given a positive integer $j, 1 \leqq j \leqq n$, define $X(j)$ as the number of elements $a_{i, j}, i \neq j$, for which $a_{i, j}>0$. Then, the corollary to Lemma 2 implies that $X(j) \geqq 1$ whenever $n>1$, for all $j$. Whenever $X(j)>1$, we may improve the result of the corollary to Lemma 2 by observing that if $1 \leqq h \leqq n-X(j)$, then $\{j\} \cup F(j) \cup \cdots \cup F^{h}(j)$ contains at least $h+X(j)$ elements. If we use this result in the proofs of Lemma 3 and Theorem 1, we obtain the following improvements.

Lemma 4. Let $k$ and $j$ be as in Lemma 3. Then, $F^{n-X(j)+k}(j)=J$.

Theorem 4. Let $A$ be as in Theorem 1. Let $X_{1}$ be the minimum of $X(j)$ for the $d$ elements $j \in J$. Let $X_{2}$ be the minimum of $X(j)$ for the remaining $n-d$ elements $j \in J$. Then,

$$
\gamma(A) \leqq 2 n-d-X_{1}-\min \left[X_{2}-1 ; n-d\right]+k .
$$


Corollary. Let $d>0$ of the diagonal elements of $A$ be positive. Then,

$$
\gamma(A) \leqq 2 n-d-X_{1}-\min \left[X_{2}-1 ; n-d\right] .
$$

A similar improvement may also be obtained for Theorem 2 .

For any non-negative irreducible matrix, we may define the (irreducible) order of $A$, denoted by $\Lambda(A)$, as the smallest positive integer $h$ such that $I+A+A^{2}+\cdots+A^{h}$ is positive, or equivalently, $\{j\} \cup \cdots \cup F^{h}(j)=J$ for each $j$. By definition of irreducibility, it is clear that $\Lambda(A) \leqq n-1$. If $\Lambda(A)$ is less than $n-1$, and the value of $\Lambda(A)$ is known, many of the preceding inequalities may be improved. We summarize how the order of $A$ may be used to sharpen respectively the results of Lemma 4, Theorem 4, and its corollary above. These results are respectively:

$$
\begin{aligned}
& F^{\min [n-X(j) ; \Lambda(A)]+k}(j)=J \\
& \gamma(A) \leqq \min \left[n-X_{1} ; \Lambda(A)\right] \\
& \quad+\min \left\{n-d-\min \left[X_{2}-1 ; n-d\right] ; \Lambda(A)\right\}+k \\
& \gamma(A) \leqq \min \left[n-X_{1} ; \Lambda(A)\right] \\
& \quad+\min \left\{n-d-\min \left[X_{2}-1 ; n-d\right] ; \Lambda(A)\right\}
\end{aligned}
$$

\section{BIBLIOGRAPHY}

1. G. Frobenius, Über Matrizen aus nicht negativen Elementen, Sitzungsberichte der Preussischen Akademie der Wissenschaften zu Berlin, 1912, pp. 456-477.

2. Helmut Wielandt, Unzerlegbare, nicht negativen Matrizen, Math. Zeit. vol. 52 (1950) pp. 642-648.

3. I. N. Herstein, $A$ note on primitive matrices, Amer. Math. Monthly vol. 61 (1954) pp. 18-20.

Los Alamos Scientific Laboratory of the University of California and

Westinghouse Electric Corporation, Bettis Plant 